Article

\title{
The Characterization of Affine Symplectic Curves in $\mathbb{R}^{4}$
}

\section{Esra Çiçek Çetin and Mehmet Bektaş *}

Department of Mathematics, Faculty of Science, Firat University, 23119 Elazı ̆̆, Turkey; esracetincicek@gmail.com

* Correspondence: mbektas@firat.edu.tr; Tel.: +90-424-237-0000

Received: 29 November 2018; Accepted: 18 January 2019; Published: 21 January 2019

check for updates

\begin{abstract}
Symplectic geometry arises as the natural geometry of phase-space in the equations of classical mechanics. In this study, we obtain new characterizations of regular symplectic curves with respect to the Frenet frame in four-dimensional symplectic space. We also give the characterizations of the symplectic circular helices as the third- and fourth-order differential equations involving the symplectic curvatures.
\end{abstract}

Keywords: symplectic curves; circular helices; symplectic curvatures; Frenet frame

\section{Introduction}

As the Riemannian geometry involves the length as the fundamental quantity, symplectic geometry involves the directed area, and contact geometry involves the twisting behavior as the fundamental quantities. Since contact geometry is always odd-dimensional and symplectic geometry is always even-dimensional, they are dual in the sense that they have many common results. Hence, studying the twisting behavior in symplectic geometry helps us to obtain connections between these two geometries.

The even-dimensional symplectic geometry has been found in numerous areas of mathematics and physics. It arises as the natural geometry of phase-space in the equations of classical mechanics, which are called Hamilton's equations, and treating mechanical problems in phase-space greatly simplifies the problem [1]. Besides, the symplectic numerical methods are known to be fast and accurate [2-5]. Symplectic geometry also arises in microlocal analysis [6-8], in time series analysis [9,10], analysis of random walks on euclidean graphs [11], and applications of Clifford algebras [12-14].

Geometrical optics has been recognized as a semi-classical limit of wave optics with a small parameter; it has nevertheless been constantly considered as a self-consistent theory for light rays, borrowing much from differential geometry and, more specifically, from Riemannian and symplectic geometries. Geometrical optics provides, indeed, a beautiful link between both previously-mentioned geometries: (i) Light travels along geodesics of an optical medium, a three-dimensional manifold whose Riemannian structure is defined by a refractive index; (ii) The set of all such geodesics is naturally endowed with the structure of four-dimensional symplectic manifolds [15,16].

The aim of this paper is to study some characterization for a special class of symplectic curves called affine symplectic helices, which are a very important tool for both physics and geometric optics. The helix is a symplectic similarity of non-symbolic full toric diversity, whereby algebraic geometry accounts for the effects of uniformity near the focus-focus singularities. The characterization of the helices in different geometries has also been studied by several researchers [17-21]. Proceeding the same way, we study symplectic regular curves, which are parameterized by the symplectic arc length and analyzed by their Frenet-type symplectic frame. In Section 2, we present the preliminaries on the symplectic geometry in terms of isometry groups and inner products. In Section 3, we give the general 
properties of affine symplectic curves in $\mathbb{R}^{4}$, which was firstly studied in [22]. Finally, in Section 4 , we present the results that we obtain on the characterizations of symplectic curves in $\mathbb{R}^{4}$ and study symplectic helices.

\section{Preliminaries}

In the following, we use similar notations and concepts as in [22].

Let $\mathbb{R}^{4}$ be endowed with standard symplectic form $\Omega$ given in global Darboux coordinates: $z=\left(x^{1}, x^{2}, y^{1}, y^{2}\right)$ by

$$
\Omega=d x^{1} \wedge d y^{1}+d x^{2} \wedge d y^{2} .
$$

Given two vector fields:

$$
\mathbf{u}=x^{1} \frac{\partial}{\partial x^{1}}+x^{2} \frac{\partial}{\partial x^{2}}+y^{1} \frac{\partial}{\partial y^{1}}+y^{2} \frac{\partial}{\partial y^{2}}
$$

and:

$$
\mathbf{v}=\xi^{1} \frac{\partial}{\partial \xi^{1}}+\xi^{2} \frac{\partial}{\partial \xi^{2}}+\omega^{1} \frac{\partial}{\partial \omega^{1}}+\omega^{2} \frac{\partial}{\partial \omega^{2}}
$$

the symplectic form (1) induces a symplectic inner product, which is a non-degenerate, skew-symmetric, bilinear form, on each fiber of tangent bundle $T \mathbb{R}^{4}$. with:

$$
<\mathbf{u}, \mathbf{v}>=\Omega(\mathbf{u}, \mathbf{v})=\sum_{i=1}^{2}\left(x^{i} \omega^{i}-y^{i} \xi^{i}\right) .
$$

The isometry group of the inner product (2) is the 10-dimensional symplectic group $S p(4)=S p(4, \mathbb{R}) \subset G L(4, \mathbb{R})$. The Lie algebra $\mathfrak{s p}(4)$ of $S p(4)$ is the vector space consisting of all $4 \times 4$ matrices of the form:

$$
\left(\begin{array}{ll}
U & V \\
W & -U^{T}
\end{array}\right)
$$

where $U, V$, and $W$ are $2 \times 2$ matrices satisfying:

$$
W=W^{T}, \quad V=V^{T} .
$$

The semi-direct product $G=S p(4, \mathbb{R}) \ltimes \mathbf{R}^{4}$ of the symplectic group by the translations is called the group of rigid symplectic motions [22]. Hence, a rigid symplectic motion acting on $z \in \mathbf{R}^{4}$ with $z \mapsto A z+b$ for $(A, b) \in S p(4, \mathbb{R})$ is an affine symplectic transformation.

Definition 1. A symplectic frame is a smooth section of the bundle of linear frames over $\mathbb{R}^{4}$, which assigns to every point $z \in \mathbb{R}^{4}$ an ordered basis of tangent vectors $a_{1}, a_{2}, a_{3}, a_{4}$ with the property that:

$$
\begin{aligned}
\left\langle a_{i}, a_{j}\right\rangle & =\left\langle a_{2+i}, a_{2+j}\right\rangle=0,1 \leq i, j \leq 2, \\
\left\langle a_{i}, a_{2+j}\right\rangle & =0,1 \leq i \neq j \leq 2 \\
\left\langle a_{i}, a_{2+i}\right\rangle & =1,1 \leq i \leq 2 .
\end{aligned}
$$

The structure equations for a symplectic frame are therefore of the form:

$$
\begin{aligned}
d a_{i} & =\sum_{k=1}^{2} w_{i k} a_{k}+\sum_{k=1}^{2} \theta_{i k} a_{2+k} \\
d a_{2+i} & =\sum_{k=1}^{2} \phi_{i k} a_{k}-\sum_{k=1}^{2} w_{k i} a_{2+k}
\end{aligned}
$$


for $1 \leq i \leq 2$. By a consequence of the conditions in (4), the one forms satisfy:

$$
\theta_{i j}=\theta j i, \quad \phi_{i j}=\phi_{j i} .
$$

\section{General Properties of Affine Symplectic Curves in $\mathbb{R}^{4}$}

We consider parametrized smooth curves $z: I \rightarrow \mathbb{R}^{4}$ defined on an open interval $I \subset \mathbf{R}$. As is customary in classical mechanics, we use the notation $\dot{z}$ to denote differentiation with respect to the parameter $t$, that is:

$$
\dot{z}=\frac{d z}{d t} .
$$

Definition 2. Let $z: I \rightarrow \mathbb{R}^{4}$ be a smooth curve. If the second-order osculating spaces of $z$ satisfy the non-degeneracy condition:

$$
<\dot{z}, \ddot{z}>\neq 0
$$

for all $t \in I$, then $z: I \rightarrow \mathbb{R}^{4}$ is called an affine symplectic regular curve.

Definition 3. Let $t_{0} \in I$. The symplectic arc length $s$ of a symplectic regular curve $z$ starting at $t_{0}$ is defined by:

$$
s(t)=\int_{t_{0}}^{t}\langle\dot{z}, \ddot{z}\rangle^{1 / 3} d t
$$

for $t \in I$.

We shall note that symplectic arc length may be negative. However, with no loss of generality, we may assume that $\langle\dot{z}, \ddot{z}>>0$ throughout the paper.

Taking the exterior differential of the (8), we obtain the symplectic arc length element as:

$$
d s=\langle\dot{z}, \ddot{z}\rangle^{1 / 3} d t
$$

In the following, primes are used to denote differentiation with respect to the symplectic arc length derivative operator (9) as:

$$
z^{\prime}=\frac{d z}{d s}
$$

Definition 4. A symplectic regular curve is parameterized by the symplectic arc length if:

$$
\langle\dot{z}, \ddot{z}\rangle=1
$$

for all $t \in I$.

Proposition 1. Every symplectic regular curve can be parameterized by the symplectic arc length.

Proposition 2. Let $z: I \rightarrow \mathbb{R}^{4}$ be a symplectic regular curve, which is parameterized by the symplectic arc length, and such that $H_{2}(s) \neq 0$. Then, the symplectic frame $\left\{a_{1}(s), a_{2}(s), a_{3}(s), a_{4}(s)\right\}$ defined along the image of $z$ satisfies the following structure equations:

$$
\begin{aligned}
& a_{1}^{\prime}(s)=a_{3}(s) \\
& a_{2}^{\prime}(s)=H_{2}(s) a_{4}(s) \\
& a_{3}^{\prime}(s)=k_{1}(s) a_{1}(s)+a_{2}(s) \\
& a_{4}^{\prime}(s)=a_{1}(s)+k_{2}(s) a_{2}(s),
\end{aligned}
$$

where $H_{2}(s), k_{1}(s), k_{2}(s)$ are symplectic curvatures of $z$. 
In general, we call the equations in (11) symplectic Frenet equations.

\section{The Characterizations of Symplectic Curves in $\mathbb{R}^{4}$}

Definition 5. Let $z: I \rightarrow \mathbb{R}^{4}$ be a symplectic regular curve, which is parameterized by the symplectic arc length, and $\left\{a_{1}(s), a_{2}(s), a_{3}(s), a_{4}(s)\right\}$ be the Frenet frame of this curve. A symplectic curve $z$ that satisfies the following condition:

$$
\frac{k_{1}(s)}{k_{2}(s)}=\text { const }
$$

is called a general helix with respect to the Frenet frame.

Example 1. Let $z: I \rightarrow \mathbb{R}^{4}$ be defined with $z(t)=\left(t, \frac{t^{2}}{2}, \frac{t^{3}}{3}, \frac{t^{3}}{3}+\frac{t^{5}}{5}\right)$. Since $\Omega(d z, d z) \neq 0$ and:

$$
\frac{k_{1}(t)}{k_{2}(t)}=\text { constant }
$$

with:

$$
d s=\langle\dot{z}, \ddot{z}\rangle^{1 / 3} d t
$$

$z$ is a symplectic polynomial helix.

Example 2. Let $z: I \rightarrow \mathbb{R}^{4}$ be defined with $z(s)=(\cosh s, 0, \sinh s, 0)$. Since $\Omega(d z, d z) \neq 0$ and:

$$
\frac{k_{1}(s)}{k_{2}(s)}=\text { constant }
$$

with:

$$
<\dot{z}, \ddot{z}>=1,
$$

$z$ is a symplectic arc length parameterized circular helix.

Definition 6. Let $z: I \rightarrow \mathbb{R}^{4}$ be a symplectic regular curve, which is parameterized by the symplectic arc length, and $\left\{a_{1}(s), a_{2}(s), a_{3}(s), a_{4}(s)\right\}$ be the Frenet frame of $z$. If both $k_{1}(s)$ and $k_{2}(s)$ are positive constants along $z$, then $z$ is called a circular helix with respect to the Frenet frame.

Theorem 1. Let $z(s)$ be a symplectic regular curve, which is parameterized by the symplectic arc length. $z(s)$ is a general helix with respect to the Frenet frame $\left\{a_{1}(s), a_{2}(s), a_{3}(s), a_{4}(s)\right\}$ such that $H_{2}(s)=$ const $\neq 0$ if and only if:

$$
a_{1}^{(i v)}(s)=\left[k_{1}^{\prime \prime}(s)+k_{1}^{2}(s)+H_{2}(s)\right] a_{1}(s)+2 k_{1}^{\prime}(s) a_{3}(s)
$$

Proof. Suppose that $z(s)$ is a general helix with respect to the Frenet frame $\left\{a_{1}(s), a_{2}(s), a_{3}(s), a_{4}(s)\right\}$. Then, from (11), we have:

$$
\begin{aligned}
a_{1}^{(i v)}(s)= & {\left[k_{1}^{\prime \prime}(s)+k_{1}^{2}(s)+H_{2}(s)\right] a_{1}(s)+\left[k_{1}(s)+\right.} \\
& \left.k_{2}(s) H_{2}(s)\right] a_{2}(s)+2 k_{1}^{\prime}(s) a_{3}(s)+H_{2}^{\prime}(s) a_{4}(s)
\end{aligned}
$$

Now, $H_{2}(s)=\operatorname{cons}(\neq 0)$, and $z(s)$ is a general helix with respect to the Frenet frame; we suppose that:

$$
\frac{k_{1}(s)}{k_{2}(s)}=-H_{2}(s)
$$

If we substitute Equation (14) in (13), we obtain (12). 
Conversely, let us assume that Equation (12) holds. We show that the curve $z(s)$ is a general helix. From (11), we obtain:

$$
a_{1}(s)=\frac{1}{k_{1}(s)}\left[a_{3}^{\prime}(s)-a_{2}(s)\right]
$$

Differentiating covariantly (15), we obtain:

$$
a_{1}^{\prime}(s)=\left(\frac{-k_{1}^{\prime}(s)}{k_{1}(s)}\right) a_{1}(s)+\left(\frac{1}{k_{1}(s)}\right)\left[a_{3}^{\prime \prime}(s)-a_{2}^{\prime}(s)\right]
$$

and so:

$$
\begin{aligned}
a_{1}^{\prime \prime}(s)= & \left(\frac{-k_{1}^{\prime}(s)}{k_{1}(s)}\right)^{\prime} a_{1}(s)+\left(\frac{-k_{1}^{\prime}(s)}{k_{1}(s)}\right) a_{3}(s) . \\
& +\left(\frac{1}{k_{1}(s)}\right)^{\prime}\left[a_{3}^{\prime \prime}(s)-a_{2}^{\prime}(s)\right]+\left(\frac{1}{k_{1}(s)}\right)\left[a_{3}^{\prime \prime \prime}-a_{2}^{\prime \prime}\right]
\end{aligned}
$$

If we use (7) in (17) and after routine calculations, we have:

$$
\frac{H_{2}^{\prime}(s)}{k_{1}(s)}=0
$$

and:

$$
\frac{-H_{2}(s) k_{2}(s)}{k_{1}(s)}=1 \text {. }
$$

Hence, we obtain $H_{2}(s)=$ const. and $\frac{k_{1}(s)}{k_{2}(s)}=$ const. This shows that $z(s)$ is a general helix.

The hypotheses of Theorem 1 and the definition of a circular helix lead us to the following corollary:

Corollary 1. Let $z(s)$ be a symplectic regular curve, which is parametrized by the symplectic arc length. $z(s)$ is a circular helix with respect to the Frenet frame $\left\{a_{1}(s), a_{2}(s), a_{3}(s), a_{4}(s)\right\}$ if and only if:

$$
a_{1}^{(i v)}(s)=\lambda a_{1}(s)
$$

where $\lambda=k_{1}^{2}(s)+H_{2}(s)=$ const.

Theorem 2. Let $z(s)$ be a symplectic regular curve, which is parametrized by the symplectic arc length. $z(s)$ is a circular helix with respect to the Frenet frame $\left\{a_{1}(s), a_{2}(s), a_{3}(s), a_{4}(s)\right\}$ if and only if:

$$
a_{4}^{\prime \prime \prime}(s)=\left(1+k_{2}^{\prime \prime}(s)-k_{1}(s) k_{2}(s)\right) a_{2}(s)+\left(2 k_{2}^{\prime}(s) H_{2}(s)\right) a_{4}(s) \text {. }
$$

Corollary 2. Let $z(s)$ be a symplectic regular curve, which is parametrized by the symplectic arc length. $z(s)$ is a general helix with respect to the Frenet frame $\left\{a_{1}(s), a_{2}(s), a_{3}(s), a_{4}(s)\right\}$ such that $H_{2}(s)=$ const $\neq 0$ if and only if:

$$
a_{4}^{\prime \prime \prime}(s)=\mu a_{2}(s)
$$

where $\mu=\left(1-k_{1}(s) k_{2}(s)\right)=$ const.

Theorem 3. Let $z(s)$ be a symplectic regular curve, which is parametrized by the symplectic arc length. $z(s)$ is a general helix with respect to the Frenet frame $\left\{a_{1}(s), a_{2}(s), a_{3}(s), a_{4}(s)\right\}$ such that $H_{2}(s)=$ const $\neq 0$ if and only if:

$$
a_{2}^{\prime \prime \prime}(s)=H_{2}(s) a_{3}(s)+H_{2}(s) K_{2}^{\prime}(s) a_{2}(s)-H_{2}(s) K_{1}(s) a_{4}(s)
$$


Corollary 3. Let $z(s)$ be a symplectic regular curve, which is parametrized by the symplectic arc length. $z(s)$ is a general helix with respect to the Frenet frame $\left\{a_{1}(s), a_{2}(s), a_{3}(s), a_{4}(s)\right\}$ such that $H_{2}(s)=$ const $\neq 0$ if and only if:

$$
a_{2}^{\prime \prime \prime}(s)=c_{1} a_{3}(s)+c_{2} a_{4}(s)
$$

$c_{1}=H_{2}(s)=$ const. and $c_{2}=H_{2}(s) K_{1}(s)=$ const.

In the rest of this section, we discuss symplectic regular curves with constant local symplectic invariants. The theorem of Cartan states that the curves with constant symplectic curvatures are precisely the orbits of the one-parameter subgroups of the affine symplectic group in four variables [23,24]. In order to determine such one-parameter subgroups, we shall directly integrate the symplectic Frenet equations of affine symplectic helices. Now, let us consider the symplectic Frenet equations given by (11) with the matrix form as:

$$
\frac{d}{d s}\left(\begin{array}{l}
a_{1} \\
a_{2} \\
a_{3} \\
a_{4}
\end{array}\right)=\left(\begin{array}{cccc}
0 & 0 & 1 & 0 \\
0 & 0 & 0 & H_{2} \\
k_{1} & 1 & 0 & 0 \\
1 & k_{2} & 0 & 0
\end{array}\right)\left(\begin{array}{l}
a_{1} \\
a_{2} \\
a_{3} \\
a_{4}
\end{array}\right)
$$

with the constant symplectic curvatures $k_{1}, k_{2}, H_{1}$. It is well known that the eigenvalues of the Frenet matrix appearing in the right-hand side of (25) are:

$$
\begin{aligned}
& \mu_{1}=\frac{1}{\sqrt{2}} \sqrt{\lambda_{1}+\sqrt{\lambda_{2}}}, \mu_{2}=-\mu_{2} \\
& \mu_{3}=\frac{1}{\sqrt{2}} \sqrt{\lambda_{1}-\sqrt{\lambda_{2}}}, \mu_{4}=-\mu_{3}
\end{aligned}
$$

where $\lambda_{1}=k_{2} H_{2}+k_{1}$ and $\lambda_{2}=\left(k_{2} H_{2}-k_{1}\right)^{2}+4 H_{2}$ [22].

Now, let us assume that $z: I \rightarrow \mathbb{R}^{4}$ is a symplectic general helix with constant positive curvatures $k_{1}, k_{2}$. Then, by Theorem $1, k_{1}=-k_{2} H_{2}$. Therefore, the eigenvalues of the Frenet matrix appearing in (25) become:

$$
\begin{aligned}
& \mu_{1}=\frac{1}{2} \sqrt[4]{\lambda}, \mu_{2}=-\mu_{2} \\
& \mu_{3}=\frac{i}{2} \sqrt{\lambda}, \mu_{4}=-\mu_{3}
\end{aligned}
$$

where $\lambda=\left(k_{1}^{2}+H_{1}\right)$ and $i=\sqrt{-1}$. Thus, if $H_{1}<-k_{1}^{2}$, then the eigenvalues are distinct complex conjugates. Similarly, if $H_{1}>-k_{1}^{2}$, then the eigenvalues are distinct reals. Depending on the two cases involving symplectic curvatures, we obtain symplectic general helices of the euclidean or hyperbolic type.

\section{Conclusions}

In our three-dimensional world, the four-dimensional Frenet formulae may seem irrelevant and useless. However, in many areas, including the classical mechanics of physics, the Frenet formulae have been applied. In this study, we study four-dimensional symplectic curves by using the Frenet frames. Our results show that a symplectic helix involves non-zero constant symplectic curvature if and only if the fourth derivative of its first component of the position vector can be described as in Equation (12). Besides, the symplectic circular helices can be characterized directly by the first component of the position vector with the fourth-order derivative.

The characterization of the symplectic helices not only depends on the first component of the position vector. The third derivatives of the second and fourth components of the position vector can be characterized as in Equations (21) and (23). Similarly, symplectic circular helices can be characterized directly by their second and fourth components of the position vector with the third-order derivatives. 
Helices are natural twisting structures; hence, studying the symplectic helix may shed light on the connection of contact and symplectic geometries.

Author Contributions: These authors contributed equally to this work.

Funding: This research received no external funding.

Conflicts of Interest: The authors declare no conflicts of interest.

\section{References}

1. Libermann, P.; Marle, C.M. Symplectic Geometry and Analytical Mechanics; Springer Netherlands: Dordrecht, The Netherlands, 1987; Volume 35.

2. Duistermaat, J.J.; Guillemin, V.W.; Hormander, L.; Vassiliev, D. Fourier Integral Operators; Birkhauser: Boston, MA, USA, 1996; Volume 2.

3. Saitoh, I.; Suzuki, Y.; Takahashi, N. The symplectic finite difference time domain method. IEEE Trans. Magn. 2001, 37, 3251-3254. [CrossRef]

4. Yang, H.W.; Song, H. Symplectic FDTD method study left-handed material electromagnetic characteristics. Opt.-Int. J. Light Electron Opt. 2013, 124, 1716-1720. [CrossRef]

5. Zhong, S.; Ran, C.; Liu, S. The Optimal Force-Gradient Symplectic Finite-Difference Time-Domain Scheme for Electromagnetic Wave Propagation. IEEE Trans. Antennas Propag. 2016, 64, 5450-5454. [CrossRef]

6. Akgüller, Ö. Poisson Bracket on Measure Chains and Emerging Poisson Manifold. Int. J. Appl. Math. Stat. 2017, 57, 56-64.

7. Bach, A. An Introduction to Semiclassical and Microlocal Analysis; Springer Science \& Business Media: New York, NY, USA, 2013.

8. Grigis, A.; Sjöstrand, J. Microlocal Analysis for Differential Operators: An Introduction; Cambridge University Press: Cambridge, UK, 1994; Volume 196.

9. Xie, H.B.; Dokos, S.; Sivakumar, B.; Mengersen, K. Symplectic geometry spectrum regression for prediction of noisy time series. Phys. Rev. E 2016, 93, 052217. [CrossRef] [PubMed]

10. Xie, H.B.; Guo, T.; Sivakumar, B.; Liew, A.W.C.; Dokos, S. Symplectic geometry spectrum analysis of nonlinear time series. Proc. R. Soc. A 2014, 470, 20140409. [CrossRef]

11. Cantarella, J.; Shonkwiler, C. The symplectic geometry of closed equilateral random walks in 3-space. Ann. Appl. Probab. 2016, 26, 549-596. [CrossRef]

12. Binz, E.; De Gosson, M.A.; Hiley, B.J. Clifford Algebras in Symplectic Geometry and Quantum Mechanics. Found. Phys. 2013, 43, 424-439. [CrossRef]

13. Crumeyrolle, A. Orthogonal and Symplectic Clifford Algebras: Spinor Structures; Springer: Dordrecht, The Netherlands, 1990; Volume 57.

14. Da Prato, G.; Zabczyk, J. Ergodicity for Infinite Dimensional Systems; Cambridge University Press: Cambridge, UK, 1996; Volume 229.

15. Duval, C.; Horvath, Z.; Horváthy, P.A. Geometrical spinoptics and the optical Hall effect. J. Geom. Phys. 2007, 57, 925-941. [CrossRef]

16. Guillemin, V.; Sternberg, S. Symplectic Techniques in Physics; Cambridge University Press: Cambridge, UK, 1990.

17. Bektaş, M. On a characterization of null helix. Bull. Inst. Math. Acad. Sin. 2001, 29, 71-78.

18. Bektaş, M. On characterizations of general helices for ruled surfaces in the pseudo-Galilean space $G_{3}^{1}$-(Part-I). J. Math. Kyoto Univ. 2004, 44, 523-528. [CrossRef]

19. Ekmekçi, N.; Hacısalihoğlu, H. On Helicec of a Lorentzian Manifold. Commun. Math. Stat. 1995, 42, 43-55.

20. Yılmaz, M.Y.; Bektaş, M. Helices of the 3-dimensional Finsler manifold. J. Adv. Math. Stud. 2009, 2, 107-113.

21. Valiquette, F. Geometric affine symplectic curve flows in $\mathbb{R}^{4}$. Differ. Geom. Its Appl. 2012, 30, 631-641. [CrossRef]

22. Kamran, N.; Olver, P.; Tenenblat, K. Local symplectic invariants for curves. Commun. Contemp. Math. 2009, 11, 165-183. [CrossRef] 
23. Fels, M.; Olver, P.J. Moving coframes: I. A practical algorithm. Acta Appl. Math. 1998, 51, 161-213. [CrossRef] 24. Fels, M.; Olver, P.J. Moving coframes: II. Regularization and theoretical foundations. Acta Appl. Math. 1999, 55, 127-208. [CrossRef]

(c)

(C) 2019 by the authors. Licensee MDPI, Basel, Switzerland. This article is an open access article distributed under the terms and conditions of the Creative Commons Attribution (CC BY) license (http:/ / creativecommons.org/licenses/by/4.0/). 